\title{
Validation of the novel Deep Reality Viewer (DRV) 3D digital stereo viewer in otology surgery
}

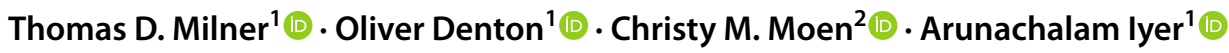

Received: 19 December 2021 / Accepted: 7 February 2022 / Published online: 22 February 2022

(c) The Author(s), under exclusive licence to Springer-Verlag GmbH Germany, part of Springer Nature 2022

\begin{abstract}
Purpose Magnification with accurate optic reproduction of the surgical field is essential in otology surgery, but current technologies are subject to specific disadvantages. This study aims to evaluate a novel 3D digital stereo viewer, the Deep Reality Viewer (DRV), in otology surgery, in comparison to both a 2D monitor and the gold standard of microscopy.

Methods In this prospective clinical research study, ENT consultants and trainees evaluated visual and practical applications of the DRV. In visual assessment, participants $(n=11)$ viewed pre-recorded in vivo mastoid exploration displayed on a $2 \mathrm{D}$ monitor and the DRV screen. In practical assessment, participants $(n=9)$ performed otology surgical tasks on a cadaveric human head using both the microscope and DRV. Face, task-specific (TSV) and global content (GCV) outcomes were assessed using 5-point Likert scale questionnaires. Construct validity was assessed separately.

Results The DRV achieved the pre-determined validation threshold of 4 for all validation parameters in both visual and practical assessment. The DRV significantly outperformed the 2D monitor in fourteen of 16 parameters. In comparison to microscopy, there was no significant difference in 13 of 16 parameters, with the DRV significantly outperforming in the remaining 3: defining anatomy (GCV), assessing middle ear anatomy (TSV) and overall TSV. Construct validity was not demonstrated for either technology.

Conclusion The DRV achieved the validation threshold for all parameters, and outperformed the 2D monitor and microscopy in several parameters. This validates the DRV for performing otological procedures, and suggests that it would be a useful alternative to the gold standard of microscopy in otology surgery.

Level of evidence N/A.
\end{abstract}

Keywords Otologic surgical procedures $\cdot$ Technology $\cdot$ Depth perception $\cdot$ Microscopy $\cdot$ Middle ear $\cdot$ 3D digital stereo

\section{Introduction}

Accurate optic reproduction of the surgical field at orders of magnification is essential in many contemporary surgical procedures. Traditionally, the gold standard in otology surgery has been microscopy, which gives natural depth perception with binocular vision, excellent illumination and magnification, and the ability to work with two hands [1].

However, use of the traditional binocular eye-piece microscope requires fixed head positioning to optimise the

Thomas D. Milner

tommilner1@doctors.org.uk

1 University Hospital Monklands, Otolaryngology, Airdrie, Scotland, UK

2 Queen Elizabeth University Hospital, Otolaryngology, Glasgow, Scotland, UK microsurgical field, which has been demonstrated to compromise operating surgeon health [2]. Additionally, many procedures in otolaryngology are aerosol-generating, and during the COVID-19 pandemic, it has become necessary to wear personal protective equipment (PPE) to minimise risk in operating. Current PPE eye protection has been recently reported not to be compatible with the use of a microscope [3]. Therefore, current use of a microscope in many otology procedures requires consideration of mitigation strategies for preservation of the safety of the surgeon.

The Deep Reality Viewer (DRV) platform integrates an optical stereo microscope with a digital stereo camera module and the 3D DRV display (as shown in Fig. 1). This novel technology offers a "glasses-free 3D experience with true depth perception, extra wide field of view, high-definition resolution and excellent subject clarity" (Vision TriTeQ ${ }^{3}$ ) [4]. Optical and digital technologies in the 3D display 
Fig. 1 A Participant performing tympanoplasty graft placement, ossiculoplasty placement and mastoid drilling on a human cadaveric head using the DRV. B DRV display

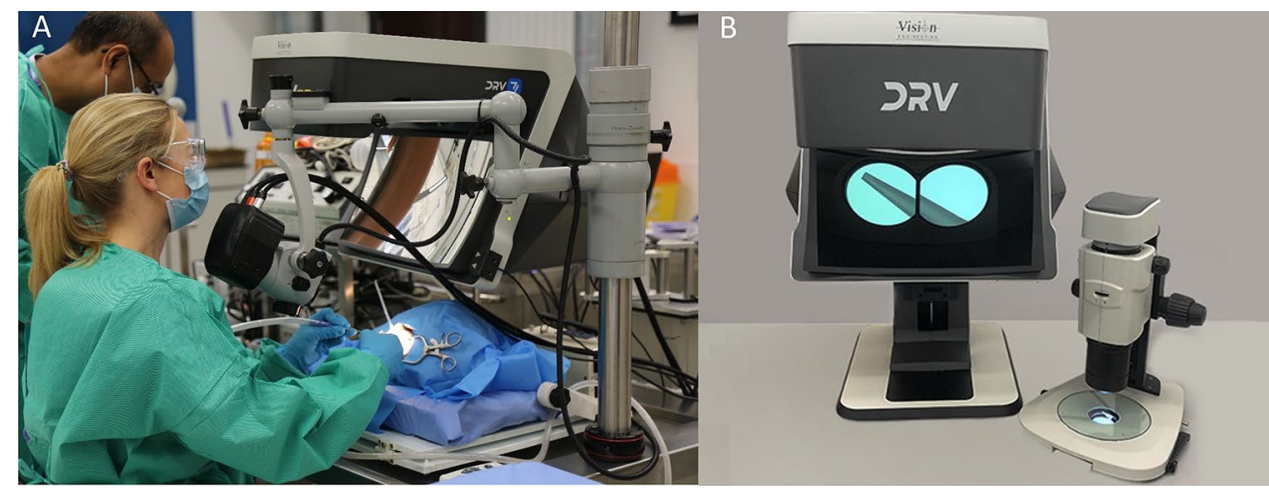

combine to project two independent optical channels, so that each eye sees the subject at a slightly different angle. This replicates natural stereo cues and depth perception, providing the surgeon with a bright, high-resolution and ghosting-free stereo image, whilst allowing the user to wear prescriptive eyewear freely. The technology comprises two LCD panels, one showing the left eye's view and the other the right, which are enclosed inside the device and, using a series of sophisticated optics, projects the stereo image to the user's eyes. The platform allows the surgeons to operate with both hands, as with the microscope, but facilitates ergonomic posture through a heads-up display setup similar to endoscopic surgery. This allows a more natural, confident and comfortable experience. The DRV platform has not yet been used intraoperatively by any surgical specialty.

This study aims to evaluate a novel 3D digital stereo viewer, the Deep Reality Viewer (DRV), in otology surgery, in comparison to both a $2 \mathrm{D}$ monitor and the gold standard of microscopy.

\section{Methods}

\section{Study Design}

This was a prospective clinical research study assessing the visual and practical applications of the DRV in comparison to microscopy and a 2D monitor display.

ENT consultants and trainees from the west of Scotland (NHS Greater Glasgow \& Clyde and NHS Lanarkshire) were invited to participate in the validation study across two arms.

Participants completed a pre-study questionnaire detailing their grade and level of experience with otological procedures. The model that each candidate assessed first was also recorded.

\section{Study Participants and Procedures}

In visual assessment, 11 ENT consultants and trainees viewed pre-recorded in vivo mastoid exploration displayed on a 2D monitor and the DRV screen (Vision Triteq Ltd, Woking, UK. https://www.visiontriteq.com). Participants were randomised in which order the videos were viewed.

In practical assessment, nine ENT consultants and trainees performed otology surgical tasks on a cadaveric human head using both the microscope (Zeiss OPMI 11) and DRV. Participants were randomised in which order the systems were used first using a random number generator. An image of the DRV system in use is attached in Fig. 1.

\section{Outcome measures}

Face, task-specific and global content outcome assessments using five-point Likert scale questionnaires were the primary outcome measure in both arms. Construct validity was assessed separately.

For face validation, all participants were asked to rate each display using five-point Likert scale ('very poor' to 'very good') in each of five face validity outcomes (Table 1), and to provide qualitative assessment of strengths and weaknesses.

Task-specific validation differed between arms of the study. In visual assessment, participants were asked to rate the statement, "This display gives effective visualization of the surgical field during mastoid exploration to allow good visualization of the following steps" using five-point Likert scale ('strongly disagree' to 'strongly agree') for each of six task-specific validity outcomes (Table 1), and to provide qualitative assessment of strengths and weaknesses. In practical assessment, participants were asked to rate the statement, "This display gives effective visualization of the surgical field during the operation to allow the following steps to be performed" using five-point Likert scale ('strongly disagree' to 'strongly agree') for each of six task-specific validity outcomes (Table 1), and to provide qualitative assessment of strengths and weaknesses.

For global content validation, all participants were asked to rate the statement, "The quality of image display effectively allowed the following actions" using five-point Likert scale 
Table 1 Validation outcomes for face, task-specific, and global content analysis in both arms of study

\begin{tabular}{llll}
\hline & Face validity & Task-specific validity & Global content validity \\
\hline Visual assessment & Image quality & Raising tympanic membrane flap & Definition of anatomy \\
& Colour contrast & Chorda tympani identification & Economy of movement \\
& Depth perception & Ossicle identification and disarticulation of incus & Operative progression \\
& Ease of viewing (e.g. lack of & Mastoid drilling & Surgical planning \\
& eye strain) & Facial nerve identification & Overall \\
& Overall & Overall & As above \\
As above & Examination under anaesthetic of external ear & \\
& & Mastoid drilling & \\
& Identification of middle ear anatomy & \\
& Tympanoplasty graft placement & \\
& Ossiculoplasty, Partial Ossicular Replacement Prosthesis & \\
\hline
\end{tabular}

('strongly disagree' to 'strongly agree') for each of five global content validity outcomes (Table 1).

Construct validity was assessed by measuring time taken to perform ossiculoplasty and tympanoplasty by microscopy and using the DRV in a cadaveric human head (practical assessment). Data collection and analysis was stratified according to surgeon experience.

\section{Analysis}

There was a pre-determined validation threshold of four out of five on the Likert scale.

In visual assessment, descriptive statistics and Wilcoxon signed rank tests were used. In practical assessment, descriptive statistics, and Wilcoxon signed rank tests were used for face, task-specific content and global content validity assessments, and paired $\mathrm{t}$ tests were used for construct validity assessments, after confirming normality of the data. Statistical analyses were conducted using $\mathrm{R}$ statistical software through R studio (version 2021.09.0) [5]. $P$ values $<0.05$ were considered significant.

\section{Compliance with ethical standards}

This study used de-identified samples of human tissue provided by the University of Glasgow Clinical Anatomy Skills Centre (CASC). This study was deemed exempt from specific approval. CASC protocols are in accordance with the ethical standards of University of Glasgow, with the Human Tissue (Scotland) Act (2006), and with the 1964 Helsinki Declaration and its later amendments or comparable ethical standards.

\section{Results}

This study had two arms, with the visual assessment having eleven participants, and the practical assessment having nine participants. Participants were either consultants or trainee surgeons (ranging from junior Foundation year two trainees to senior ST8 Specialist registrars).

The visual assessment had four consultants and seven trainees participating. The practical assessment had five consultants and four trainees.

\section{D screen vs 3D DRV: visual assessment}

Participants compared the face, task-specific content and global content validity between the 3D DRV viewer and a 2D display screen when observing a simulated mastoidectomy. All parameters for 3D viewer achieved the validation threshold of 4 for face, task-specific and global content assessments. The mean overall scores for the $3 \mathrm{D}$ viewer were 4.7, 4.9 and 4.9, for face, task-specific and global content validity, respectively.

The 2D viewer achieved lower validation scores and failed to reach the equivalent threshold of 4 set for the 3D viewer when assessing 'colour contrast', 'depth perception', 'mastoid drilling', 'economy of movement', 'operative progression' and 'surgical planning'. In addition, overall face validation scores were below the required threshold (Likert score $=3.9$ ), while overall validation scores for task-specific and global content validity were 4.2 and 4, respectively.

In direct comparison to the $2 \mathrm{D}$ viewer, the $3 \mathrm{D}$ viewer significantly outperformed it in all parameters except 'ease of viewing', and 'raising a posterior tympanomeatal flap'.

Data are represented in bar charts for face (Fig. 2A), task-specific content (Fig. 2B) and global content validity (Fig. 2C).

Of note, there was no difference between trainees and consultants in terms of their opinion on the $3 \mathrm{D}$ viewer.

\section{Microscope vs. 3D DRV: practical assessment}

The 3D display achieved the validation threshold of 4 for all face, task-specific and global content parameters assessed. 


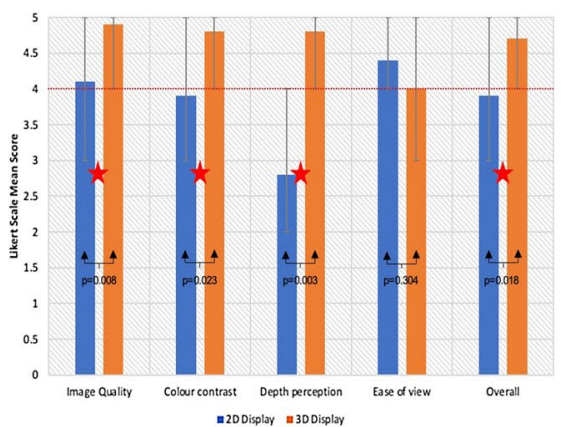

A: Face Validity

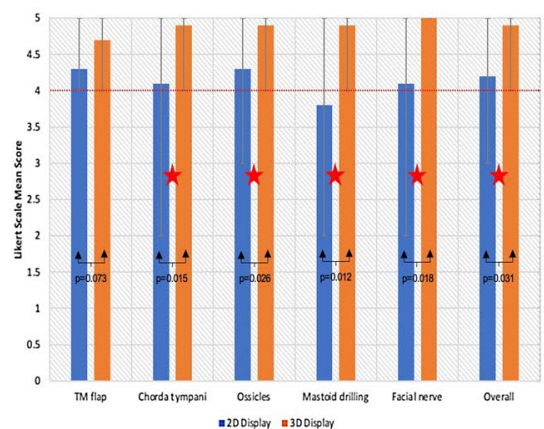

B: Task-Specific Content Validity

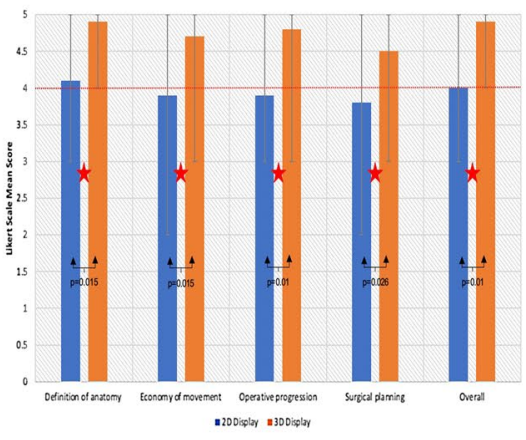

C: Global Content Validity
Fig. 2 Visual assessment outcomes for face validity (A), task-specific content validity $(\mathbf{B})$ and global content validity $(\mathbf{C})$ outcomes: 2D vs. 3D DRV display demonstrating mean Likert scale scores. Error bars

In comparison to the microscope, there was no significant difference in face validity outcomes using the 3D DRV: image quality, colour contrast, depth perception, ease of viewing and overall face validity assessment were all deemed to be of equal quality in comparison to the microscope (Fig. 3A). Of note, the 3D viewer scored higher in all parameters, however, no statistical significance was demonstrated.

In task-specific validity outcomes, the $3 \mathrm{D}$ viewer was significantly better for 'identifying middle ear anatomy' $($ microscope mean $=4.2,3 \mathrm{D}$ mean $=4.9, W=0, p=0.02)$ and 'overall' (microscope mean $=4.3,3 \mathrm{D}$ mean $=4.9, W=0$, $p=0.037$ ) (Fig. 3B). The 3D viewer performed better in other tasks 'EUA of EAC', 'mastoid drilling' and 'ossiculoplasty PORP placement' although these differences were not statistically significant. No difference was found in tympanoplasty graft placement between microscope and 3D viewer.

In global content validity outcomes, the $3 \mathrm{D}$ viewer was significantly better at 'defining anatomy' (microscope mean $=4.3,3 \mathrm{D}$ mean $=4.9, W=0, p=0.037$ ) (Fig. 3C). The $3 \mathrm{D}$ viewer also scored higher for 'economy of movement', represent the maximum to minimum values. The dotted red line represents the validation threshold. Significant results are marked with a red asterisk

'operative progression', 'surgical planning' and 'overall', although these were not statistically significant.

Both tympanoplasty graft placement and ossiculoplasty PORP placement procedures were timed for each participant. While both of these procedures were performed more quickly with the microscope in comparison to the DRV, these outcomes were not statistically significant.

\section{Construct validity assessment}

Construct validity aims to determine if the assessment of the DRV differed according to level of experience of the participant. The timed assessments demonstrated no difference between trainees and consultants in terms of speed performing tympanoplasty either using the microscope (trainees $=86.5 \mathrm{~s}$, consultants $=63.8 \mathrm{~s}, t=0.87$, $d f=3.38, p=0.44$ ), or using the DRV (trainees $=116 \mathrm{~s}$, consultants $=95.2 \mathrm{~s}, t=0.43, d f=4.38, p=0.69)$. Similarly, speed performing ossiculoplasty using the microscope $($ trainees $=104.5 \mathrm{~s}$, consultants $=87.8 \mathrm{~s}, t=0.78$, $d f=6.38, p=0.46$ ), and the DRV (trainees $=137.25 \mathrm{~s}$,

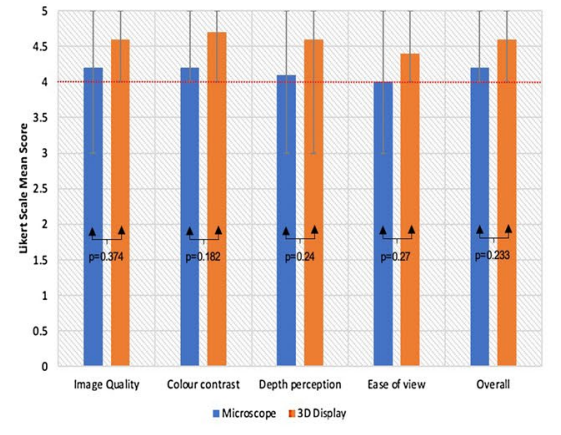

A: Face Validity

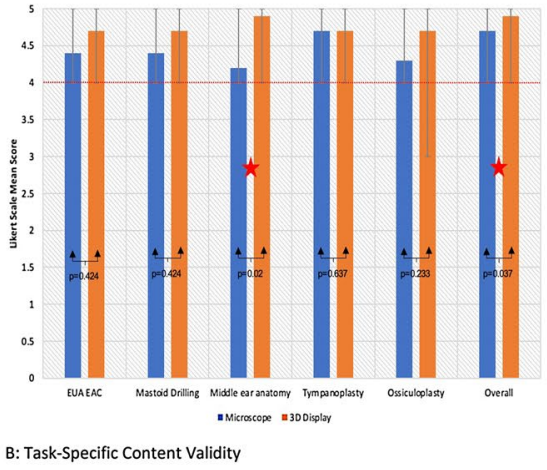

scores. Error bars represent the maximum to minimum values. The dotted red line represents the validation threshold. Significant results are marked with a red asterisk 
consultants $=78.2 \mathrm{~s}, t=1.02, d f=3.31, p=0.37$ ), were also not significantly different.

In addition, when analysing questionnaire Likert scale scoring outcomes, there was no significant difference between trainees and consultants in terms of their assessment of the DRV. Construct validity outcomes are demonstrated in Fig. 4.

\section{Discussion}

This study has demonstrated superiority of the DRV system in comparison to the traditional microscope view in several key parameters when performing simulated otological procedures. It was found to be superior to a 2D screen when visualising a procedure in almost all domains. In comparison to microscope use during a practical assessment, the DRV was rated higher in all domains measured. It was deemed particularly useful for defining anatomy (task-specific-defining middle ear anatomy, global content-defining anatomy), and improving overall progression of operative steps (overall task-specific evaluation), having demonstrated significance in these parameters.

The improved performance demonstrated by the 3D DRV in this study, particularly in comparison to the $2 \mathrm{D}$ visual assessment, and when defining anatomy, demonstrates the importance of 3D visual assessment intraoperatively. Preservation of $3 \mathrm{D}$ visualisation of the surgical field is essential in establishing an effective alternative to traditional microscopy. There is clear evidence of superior operator performance in comparison to two-dimensional (2D) visualisation. A 2018 meta-analysis showed significantly reduced operating time, less blood loss, and shorter hospital stays with $3 \mathrm{D}$ versus $2 \mathrm{D}$ endoscopic laparoscopic or thoracoscopic surgery [6]. Wilhelm et al. [7] used electromagnetic instrument pathway tracking to demonstrate an approximate $20 \%$ improvement in task performance for surgeons using a 3D compared to 2D display during laparoscopy [7]. Two systemic reviews comparing 3D and 2D laparoscopy suggest improved speed, and critically, reduced incidence of performance error with 3D technologies [8,9]. Similarly, the benefit of 3D operating has been demonstrated within the field of ENT. In simulated functional endoscopic sinus surgery, 3D endoscopy resulted in improved operative speed, and a reduction in operative errors [10]. Similarly, in endoscopic ear surgery, a developing field within ENT, 3D endoscopy has demonstrated improved perceived stereoscopic vision and depth perception in comparison to 2D endoscopy [11, 12].

There were other important results found. No significant difference was found between the time taken to perform tasks on the DRV compared to the microscope. This is an important finding, as this was the first time any of the study participants had used the DRV, whereas all had used a microscope before. This suggests that DRV allows users to quickly become accustomed to using it. In addition to this, there was no difference found between the time it took consultants to complete the procedure compared to trainees when using the DRV. The range in experience and operative skill amongst our cohort is beneficial to this study, because it highlights the applicability of this technology to novice trainees and experts alike. It is important to note that these tasks only assessed the physical act of carrying out the procedure and did not assess operative planning as a whole.
Fig. 4 Comparison of time to completion of tympanoplasty and ossiculoplasty using a microscope and the 3D display, respectively.

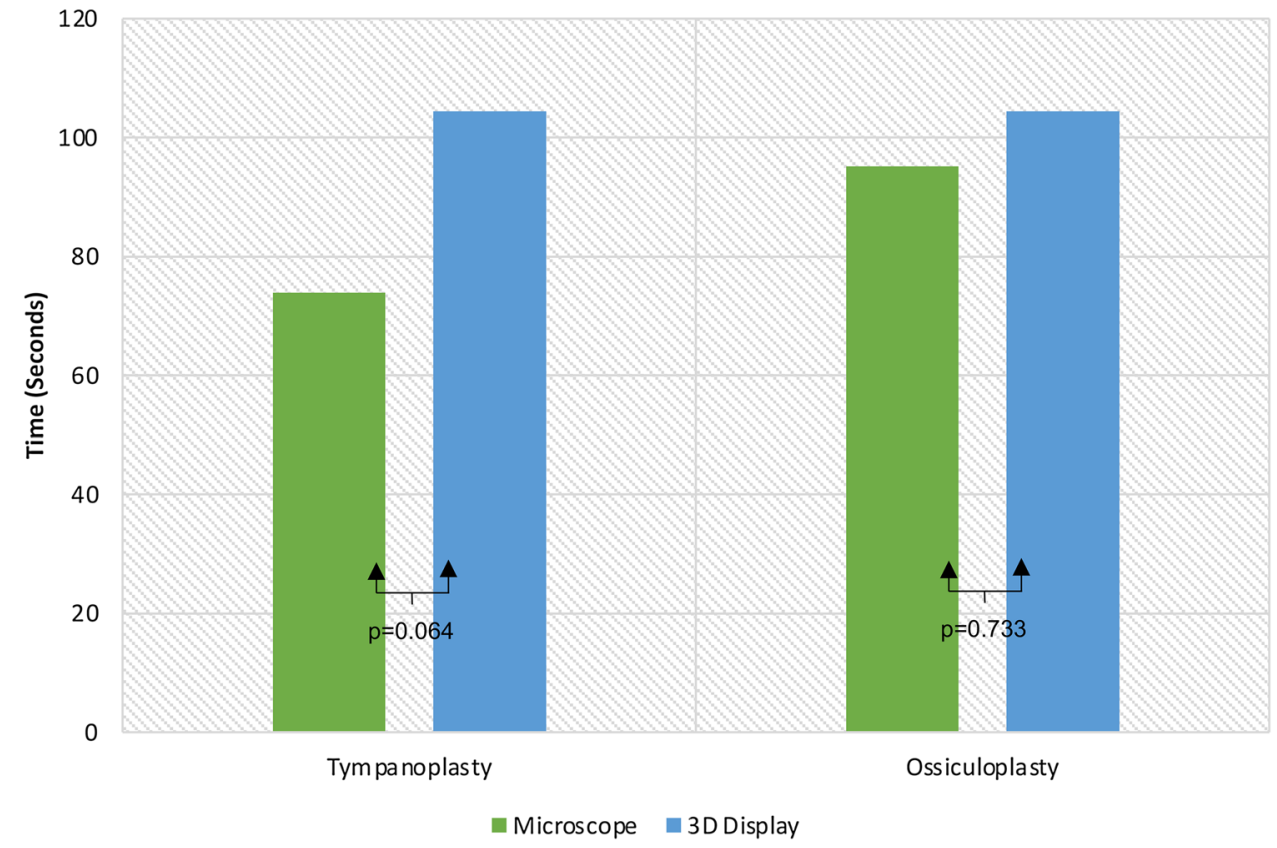


Felisati et al. [13] also identified a very rapid learning curve, with surgeons quickly developing satisfaction with a 3D system during endoscopic skull base surgery [13].

Although not specifically assessed in this study, a device must allow its user to carry out tasks repeatedly without causing injury to the user. Technologies facilitating headsup surgery aim to avoid health issues associated with use of binocular eye-piece microscopes, particularly in specialties which rely heavily on operating microscopes. A 2015 survey of 518 UK consultant ophthalmologists reported that $62.4 \%$ of respondents had neck or back pain, and $78.7 \%$ found that operating exacerbated their pain. Of those with pain, $57.1 \%$ had used non-steroidal anti-inflammatory drugs (NSAIDs), $40 \%$ had received physiotherapy, and $4.9 \%$ had undergone surgery [2]. A recent comparative assessment of ergonomic experience in heads-up display (HUD) versus conventional microscopy showed a significant reduction in pain and discomfort with increased use of HUD, and identified heads-up operating as an important tool for surgeon wellness [14]. This replicated findings of a 2020 systematic review [15].

$3 \mathrm{D}$ endoscopy is currently the only $3 \mathrm{D}$ alternative to microscopy in otology surgery but has a number of limitations both in instrument design and existing viewing technologies. The primary shortfall of endoscopy is that it requires dissection with one hand without suction, which is more challenging [1]. It also requires user experience in fulcrum effect of the endoscope, with a learning curve of approximately 70 cases evident $[11,16]$. In 3D endoscopy, there is the added requirement to achieve and maintain a "stereoscopic window" or "comfort zone" to gain maximum benefit [17]. Current heads-up displays also rely on the use of polarising glasses, which are awkward to wear with prescription eyewear, and can result in deterioration of image quality. Additionally, a proportion of surgeons have been reported to suffer from side effects of incongruency in digital stereopsis (fatigue, dizziness, headache, eye strain) [17, 18]. Recent studies in otology have suggested exoscopes may be a useful alternative to endoscopy in heads-up surgery, with the added benefit of avoiding a 'one handed' approach. However, these report significantly worse visibility in deeper parts of the surgical field, or requirement to wear 3D eyewear $[19,20]$. The use of DRV removes all of these barriers, providing an ergonomic heads-up display, with dual-hand operating, without the need for 3D glasses and the consequent eye strain.

The main limitation of our study was its small cohort size, reducing the statistical power of the analyses. However, smaller cohort sizes are common in simulation-based studies such as this one, and the study still demonstrated significance in several fields. Our study also only analysed two procedural steps within a tympanoplasty procedure and results, therefore, may not be able to be extrapolated to entire otological operations.

\section{Conclusion}

The 3D DRV achieved the validation threshold for all parameters assessed, and it outperformed both the $2 \mathrm{D}$ screen as well as the microscope in several of these validation parameters. This indicates that the DRV is a safe, and in some criteria, a more effective operative tool for performing otological procedures. It also suggests that it would be a useful alternative to the gold standard of microscopy in otology surgery. This study supports the assessment of the DRV system in the operating theatre for otology surgery.

Author contributions Contributions to article development are detailed below: TDM and AI obtained the data and performed the preliminary analysis. TDM performed the main analysis. TDM and OD wrote the Introduction, Methods. TDM, CMM and OD wrote the Discussion. TDM and CMM wrote the Results and Conclusion. All authors approved the final draft of the paper.

Funding Vision engineering has provided financial aid through funding for the anatomy laboratory, and through loan of the DRV system.

\section{Declarations}

Conflict of interest Financial interests: The senior author, Professor Arunachalam Iyer, conducts consultancy work for Vision engineering. The remaining authors have no financial interests to declare. Nonfinancial interests: None of the authors have non-financial interests to declare.

Ethical approval Approval to conduct this study was provided by the University of Glasgow Clinical Anatomy Skills Centre (CASC).

Informed consent Informed consent was obtained from all individuals involved in the study. The authors confirm that informed consent for publication was provided by the individuals contained in Fig. 1

\section{References}

1. Kozin ED, Lee DJ (2017) Basic principles of endoscopic ear surgery. Oper Tech Otolaryngol Head Neck Surg 28:2-10. https:// doi.org/10.1016/j.otot.2017.01.001

2. Hyer JN, Lee RM, Chowdhury HR et al (2015) National survey of back and neck pain amongst consultant ophthalmologists in the United Kingdom. Int Ophthalmol 35:769-775. https://doi.org/10. 1007/s10792-015-0036-z

3. Iyer A, Tikka T, Calder N et al (2021) Effect of personal protection equipment (PPE) and the distance from the eye piece of surgical microscope on the field of vision; an experimental study. Otol Neurotol 42:606-613. https://doi.org/10.1097/MAO.00000 00000002989

4. Vision Engineering DRV-Z1 (2021) In: Vision Engineering. https://www.visioneng.com/products/3d-digital-inspection/drvz1/. Accessed 26 Nov 2021

5. R Core Team (2021). R: a language and environment for statistical computing. R Foundation for Statistical Computing, Vienna, Austria. https://www.R-project.org/ 
6. Liang H, Liang $\mathrm{W}$, Lei $\mathrm{Z}$ et al (2018) Three-dimensional versus two-dimensional video-assisted endoscopic surgery: a meta-analysis of clinical data. World J Surg 42:3658-3668. https://doi.org/ 10.1007/s00268-018-4681-z

7. Wilhelm D, Reiser S, Kohn N et al (2014) Comparative evaluation of HD 2D/3D laparoscopic monitors and benchmarking to a theoretically ideal 3D pseudodisplay: even well-experienced laparoscopists perform better with 3D. Surg Endosc 28:2387-2397. https://doi.org/10.1007/s00464-014-3487-9

8. Sørensen SMD, Savran MM, Konge L, Bjerrum F (2016) Threedimensional versus two-dimensional vision in laparoscopy: a systematic review. Surg Endosc 30:11-23. https://doi.org/10.1007/ s00464-015-4189-7

9. Fergo C, Burcharth J, Pommergaard H-C et al (2017) Threedimensional laparoscopy vs 2-dimensional laparoscopy with high-definition technology for abdominal surgery: a systematic review. Am J Surg 213:159-170. https://doi.org/10.1016/j.amjsu rg.2016.07.030

10. Bickerton R, Nassimizadeh A-K, Ahmed S (2019) Three-dimensional endoscopy: The future of nasoendoscopic training. Laryngoscope 129:1280-1285. https://doi.org/10.1002/lary.27812

11. Fradeani D, Milner TD, Iyer A (2021) Learning curve in endoscopic tympanoplasties: A prospective study based on outcomes of 141 cases. Clin Otolaryngol 46:888-892. https://doi.org/10. 1111/coa.13746

12. Chen C-K, Hsieh L-C, Hsu T-H (2018) Novel three-dimensional image system for endoscopic ear surgery. Eur Arch Otorhinolaryngol 275:2933-2939. https://doi.org/10.1007/s00405-018-5153-7

13. Felisati G, Pipolo C, Maccari A et al (2013) Transnasal 3D endoscopic skull base surgery: questionnaire-based analysis of the learning curve in 52 procedures. Eur Arch Otorhinolaryngol 270:2249-2253. https://doi.org/10.1007/s00405-012-2328-5

14. Weinstock RJ, Ainslie-Garcia MH, Ferko NC et al (2021) Comparative assessment of ergonomic experience with heads-up display and conventional surgical microscope in the operating room. Clin Ophthalmol 15:347-356. https://doi.org/10.2147/ OPTH.S292152

15. Morris L, Wiley W, Sandwick V et al (2020) Evaluating the ergonomic outcomes of digitally assisted vitreo-retinal surgery (DAVS): a systematic literature review. Value Health 23:S740. https://doi.org/10.1016/j.jval.2020.08.2003

16. Yu S-T, Han P, Liang F et al (2017) Three-dimensional versus two-dimensional endoscopic-assisted thyroidectomy via the anterior chest approach: a preliminary report. Surg Endosc 31:41944200. https://doi.org/10.1007/s00464-017-5477-1

17. Singh A, Saraiya R (2013) Three-dimensional endoscopy in sinus surgery. Curr Opin Otolaryngol Head Neck Surg 21:3-10. https:// doi.org/10.1097/MOO.0b013e32835bf58c

18. Ko JKY, Li RHW, Cheung VYT (2015) Two-dimensional versus three-dimensional laparoscopy: evaluation of physicians' performance and preference using a pelvic trainer. J Minim Invasive Gynecol 22:421-427. https://doi.org/10.1016/j.jmig.2014.11.007

19. Wierzbicka M, Szyfter W, Greczka G, Gawęcki W (2021) Otosurgery with the high-definition three-dimensional (3D) exoscope: advantages and disadvantages. J Clin Med 10:777. https://doi.org/ $10.3390 / \mathrm{jcm} 10040777$

20. Ally M, Kullar P, Mochloulis G, Vijendren A (2021) Using a $4 \mathrm{~K}$ three-dimensional exoscope system (Vitom 3D) for mastoid surgery during the coronavirus disease 2019 pandemic. J Laryngol Otol 135:273-275. https://doi.org/10.1017/S002221512100044X

Publisher's Note Springer Nature remains neutral with regard to jurisdictional claims in published maps and institutional affiliations. 